\title{
SOME REMARKS ON GROWTH AND UNIQUENESS IN THERMOELASTICITY
}

\author{
RAMÓN QUINTANILLA
}

Received 13 August 2001 and in revised form 16 June 2002

\begin{abstract}
We use the Lagrange identity method and the logarithmic convexity to obtain uniqueness and exponential growth of solutions in the thermoelasticity of type III and thermoelasticity without energy dissipation. As this is not the first contribution of this kind in this theory, it is worth remarking that the assumptions we use here are different from those used in other previous contributions. We assume that the elasticity tensor is positive semidefinite, but we allow that the constitutive tensor of the entropy flux vector $\left(k_{i j}\right)$, which is a characteristic tensor in this theory, is not sign-definite. The Lagrange identity method is used to obtain uniqueness in the context of the thermoelasticity of type III. The fundamental key to obtain exponential growth in the thermoelasticity without energy dissipation is the use of a new functional. This functional is inspired in that it is used when the elasticity tensor is not sign-definite, but $\left(k_{i j}\right)$ is positive definite.
\end{abstract}

2000 Mathematics Subject Classification: 35Q72, 74F05, 74H25.

1. Introduction. The usual theory of heat conduction based on the Fourier law allows the phenomena of the infinite diffusion velocity which is not well accepted from a physical or engineering point of view. The articles of Dreyer and Struchtrup [5] and Caviglia et al. [2] provide an extensive survey of work on experiments involving the propagation of heat as a thermal wave. They report instances where the phenomena of second sound has been observed in several kind of materials. This kind of fact has provoked an explosion of activity in the field of heat propagation. Extensive reviews on the second sound theories are the work of Chandrasekharaiah [3] and the books of Jou et al. [12], Müller and Ruggeri [14].

In the more recent surveys of Chandrasekharaiah [4] and Hetnarski and Ignaczack [11], the theory proposed by Green and Naghdi [7, 8, 9, 10] is considered as an alternative formulation of the propagation of heat. This theory is developed in a rational way to produce a fully consistent theory which is capable of incorporating thermal pulse transmission in a very logical manner. They make use of a general entropy balance rather than an entropy inequality. The development is quite general and the characterization of material response for the thermal phenomena is based on three types of constitutive functions, that is, they labeled of types I, II, and III. When the theory of type I is linearised, 
the parabolic equation of the heat conduction arises. The theory of type II (is a limiting case of the type III) does not admit energy dissipation. This theory is usually called "without energy dissipation."

The relevant equations of anisotropic inhomogeneous thermoelasticity of type II with a center of symmetry are

$$
\begin{gathered}
\rho \ddot{u}_{i}=\left(a_{i j k h} u_{h, k}\right)_{, j}-\left(a_{i j} \theta\right)_{, j}+\rho f_{i}, \\
c \ddot{\theta}=-a_{i j} \ddot{u}_{i, j}+\left(k_{i j} \theta_{, j}\right)_{, i}+\rho r,
\end{gathered}
$$

where $\left(u_{i}\right)$ is the displacement vector field and $\theta$ is the temperature field. Standard notation is employed throughout the paper, and a superposed dot denotes $\partial / \partial t$. The quantities $\rho, f_{i}$, and $r$ are density, body, and heat supply, while $c$ is the specific heat. The tensor $a_{i j}$ is the coupling tensor, and we assume that the elasticity tensor $\left(a_{i j k l}\right)$ and the constitutive tensor of the entropy flux vector $\left(k_{i j}\right)$ satisfy the symmetry relations

$$
a_{i j k l}=a_{l k j i}, \quad k_{i j}=k_{j i}
$$

In a recent paper, Quintanilla and Straughan [17] proved the uniqueness and exponential growth of the solutions of system (1.1) and (1.2) whenever the tensor $\left(k_{i j}\right)$ is positive meanwhile the density and the specific heat are positive, but the elasticity coefficients are not sign-definite. In fact, the growth results were extended to the thermoelasticity of type III in [15]. We also recall the results concerning the existence obtained in [16]. The aim of this paper is to obtain results similar to those presented in [17] in the case that the density and the specific heat are positive, the elasticity tensor is positive semidefinite, but the tensor $\left(k_{i j}\right)$ is not sign-definite. The method follows the ideas of [17], after seeing the similar game of the variables $\left(u_{i}\right)$ and $\theta$ in the system. We can see this paper as a continuation and a consequence of the ideas used in [17].

Let $B$ be a bounded domain in the three-dimensional Euclidean space with boundary $\partial B$ smooth enough to allow applications of the divergence theorem. The set of (1.1) and (1.2) (or (1.6) and (1.7) see below) hold on $B \times(0, T)$ for some time $T(\leq \infty)$, and we consider boundary and initial conditions of the form

$$
\begin{gathered}
u_{i}(\mathbf{x}, t)=u_{i}^{*}(\mathbf{x}, t), \quad \theta(\mathbf{x}, t)=\theta^{*}(\mathbf{x}, t), \quad \mathbf{x} \in \partial B, \\
u_{i}(\mathbf{x}, 0)=u_{i}^{0}(\mathbf{x}), \quad \dot{u}_{i}(\mathbf{x}, 0)=v_{i}^{0}(\mathbf{x}), \quad \theta(\mathbf{x}, 0)=\theta^{0}(\mathbf{x}), \quad \dot{\theta}(\mathbf{x}, 0)=\eta^{0}(\mathbf{x}) .
\end{gathered}
$$

In Section 2, we use the Lagrange identity method to obtain a uniqueness result for the thermoelasticity of type III. It will be worth recalling that the system of 
equations governing the thermoelasticity of type III with a center of symmetry is

$$
\begin{gathered}
\rho \ddot{u}_{i}=\left(a_{i j k h} u_{h, k}\right)_{, j}-\left(a_{i j} \theta\right)_{, j}+\rho f_{i}, \\
c \ddot{\theta}=-a_{i j} \ddot{u}_{i, j}+\left(k_{i j} \theta_{, j}\right)_{, i}+\left(b_{i j} \dot{\theta}_{, j}\right)_{, i}+\rho r,
\end{gathered}
$$

where $b_{i j}=b_{j i}$. In Section 3, we show how the logarithmic convexity argument may be adapted to derive exponential growth for a measure of the solutions in the context of the thermoelasticity without energy dissipation.

2. Thermoelasticity of type III: uniqueness. The aim of this section is to obtain a uniqueness result for the solutions of system (1.6) and (1.7). From now on, we assume that the functions $\rho$ and $c$ are greater than or equal to a positive constant and that the following inequality

$$
a_{i j k h} \zeta_{i j} \zeta_{h k} \geq 0, \text { for all tensor }\left(\zeta_{i j}\right) \text {, }
$$

is satisfied. We also assume (in this section) that the tensor $\left(b_{i j}\right)$ satisfies the inequality

$$
b_{i j} \zeta_{i} \zeta_{j} \geq 0, \text { for all vector }\left(\zeta_{i}\right)
$$

and we also assume (1.3).

The proof is a direct consequence of two equalities obtained in ([17], see equations (3.9), (3.10)), but we give a brief description of it.

To consider the uniqueness question, it is sufficient to see that the null solution is the only solution that satisfies the system

$$
\begin{gathered}
\rho \ddot{u}_{i}=\left(a_{i j k h} u_{h, k}\right)_{, j}-\left(a_{i j} \theta\right)_{, j}, \\
c \ddot{\theta}=-a_{i j} \ddot{u}_{i, j}+\left(k_{i j} \theta_{, j}\right)_{, i}+\left(b_{i j} \dot{\theta}_{, j}\right)_{, i},
\end{gathered}
$$

with homogeneous boundary and initial conditions.

It is worth remarking that (2.4) can be written as

$$
c \ddot{\tau}=-a_{i j} \dot{u}_{i, j}+\left(k_{i j} \tau_{, j}\right)_{, i}+\left(b_{i j} \theta_{, j}\right)_{, i},
$$

where

$$
\tau(\mathbf{x}, t)=\int_{0}^{t} \theta(\mathbf{x}, s) d s .
$$


Some arguments related with the Lagrange identity method lead to the following identity ([17], see equation (3.9)):

$$
\int_{B}\left(\rho \dot{u}_{i} \dot{u}_{i}+k_{i j} \tau_{, i} \tau_{, j}-a_{i j k h} u_{i, j} u_{h, k}-c \theta^{2}\right) d V=0
$$

Multiplying (2.3) by $\dot{u}_{i}$ and (2.5) by $\theta$, and then integrating over $B$ with the aid of the boundary conditions, we find the energy equation

$$
E_{1}(t)=\frac{1}{2} \int_{B}\left(\rho \dot{u}_{i} \dot{u}_{i}+a_{i j k h} u_{i, j} u_{h, k}+c \theta^{2}+k_{i j} \tau_{, i} \tau_{, j}+2 \int_{0}^{t} b_{i j} \theta_{, i} \theta_{, j} d s\right) d V=0
$$

By a combination of (2.7) and (2.8), we find that

$$
\int_{B}\left(c \theta^{2}+a_{i j k h} u_{i, j} u_{h, k}+\int_{0}^{t} b_{i j} \theta_{, i} \theta_{, j} d s\right) d V=0 .
$$

We immediately deduce that $\theta \equiv 0$. Now, the vector field $\left(u_{i}\right)$ satisfies the system of the isothermal elasticity with homogeneous initial and boundary conditions. Thus, $\left(u_{i}\right) \equiv 0$, and so uniqueness follows.

REMARK 2.1. In [17], a uniqueness theorem under alternative conditions on the constitutive tensors was established. We believe that the remarks proposed at the end of Section 2 of [17] have a natural counterpart under the assumptions proposed in this section. We could employ mixed boundary conditions involving conditions (1.4) on part of the boundary with prescribed traction and entropy flux on the remainder. It also seems possible to modify previous arguments to consider the case of unbounded domains.

REMARK 2.2. If we take into account the results of [17], we may conclude the uniqueness of solutions of system (1.1) and (1.2) whenever at least one of the tensors $\left(\left(a_{i j k l}\right)\right.$ and $\left.\left(k_{i j}\right)\right)$ is positive (see $(2.1)$ and $\left.[17,(2.1)]\right)$. It is natural to ask about uniqueness in the case that neither of the tensors satisfies the above condition.

It is worth recalling that Green [6] obtained a uniqueness result in the context of the thermoelastic theory of Green-Lindsay using the Lagrange identity method.

3. Thermoelasticity of type II: exponential growth. In this section, we derive some exponential growth results for the solutions of the equations of the thermoelasticity of type II.

Let $\left(u_{i}, \theta\right)$ be a solution of (2.3) and (2.4) (with $\left.b_{i j} \equiv 0\right)$ for the homogeneous boundary conditions. We also assume that $\left(u_{i}, \theta\right)$ satisfies the initial data (1.5). The key is to define a suitable functional to which the logarithmic convexity is applicable. 
In this situation, an energy equation is

$$
E_{2}(t) \equiv \frac{1}{2} \int_{B}\left(\rho \ddot{u}_{i} \ddot{u}_{i}+a_{i j k h} \dot{u}_{i, j} \dot{u}_{h, k}+c(\dot{\theta})^{2}+k_{i j} \theta_{, i} \theta_{, j}\right) d V=E_{2}(0)
$$

We now define the functional

$$
G_{\omega, t_{0}}(t)=\int_{B}\left(c \theta^{2}+a_{i j k h} u_{i, j} u_{h, k}\right) d V+\omega\left(t+t_{0}\right)^{2},
$$

where $\omega$ and $t_{0}$ are constants to be selected. The addition of the term $\omega\left(t+t_{0}\right)^{2}$ is an idea of Knops and Payne [13] in the context of isothermal elasticity. By differentiation, we see that

$$
\begin{gathered}
G_{\omega, t_{0}}^{\prime}(t)=2 \int_{B}\left(c \theta \dot{\theta}+a_{i j k h} u_{i, j} \dot{u}_{h, k}\right) d V+2 \omega\left(t+t_{0}\right), \\
G_{\omega, t_{0}}^{\prime \prime}(t)=2 \int_{B}\left(c(\dot{\theta})^{2}+a_{i j k h} \dot{u}_{i, j} \dot{u}_{h, k}+c \theta \ddot{\theta}+a_{i j k h} u_{i, j} \ddot{u}_{h, k}\right) d V+2 \omega .
\end{gathered}
$$

We also have

$$
\begin{gathered}
\int_{B}\left(\rho \ddot{u}_{i} \ddot{u}_{i}+a_{i j k h} u_{h, k} \ddot{u}_{i, j}\right) d V=\int_{B} a_{i j} \ddot{u}_{i, j} \theta d V, \\
\int_{B}\left(c \theta \ddot{\theta}+k_{i j} \theta_{, i} \theta_{, j}\right) d V=-\int_{B} a_{i j} \ddot{u}_{i, j} \theta d V .
\end{gathered}
$$

Upon addition,

$$
\int_{B}\left(c \theta \ddot{\theta}+a_{i j k h} u_{h, k} \ddot{u}_{i, j}\right) d V=-\int_{B}\left(\rho \ddot{u}_{i} \ddot{u}_{i}+k_{i j} \theta_{, i} \theta_{, j}\right) d V .
$$

After the use of the previous inequalities, it is not difficult to see that

$$
\begin{aligned}
G_{\omega, t_{0}} & G_{\omega, t_{0}}^{\prime \prime}-\left(G_{\omega, t_{0}}^{\prime}\right)^{2} \\
= & 4\left(\int_{B}\left(c \theta^{2}+a_{i j k h} u_{h, k} u_{i, j}\right) d V+\omega\left(t+t_{0}\right)^{2}\right) \\
& \times\left(\int_{B}\left(c(\dot{\theta})^{2}+a_{i j k h} \dot{u}_{h, k} \dot{u}_{i, j}\right) d V+\omega\right) \\
& -4\left(\int_{B}\left(c \theta \dot{\theta}+a_{i j k h} u_{h, k} \dot{u}_{i, j}\right) d V+\omega\left(t+t_{0}\right)\right)^{2}-2 G_{\omega, t_{0}}\left(2 E_{2}(0)+\omega\right) .
\end{aligned}
$$

Since the Cauchy-Schwarz inequality, the following estimate follows

$$
G_{\omega, t_{0}} G_{\omega, t_{0}}^{\prime \prime}-\left(G_{\omega, t_{0}}^{\prime}\right)^{2} \geq-2 G_{\omega, t_{0}}\left(2 E_{2}(0)+\omega\right) .
$$

Now, we may state the following result. 
THEOREM 3.1. If (a) $E_{2}(0)<0$; or (b) $E_{2}(0)=0$ and $G_{\omega, t_{0}}^{\prime}(0)>0$; or (c) $E_{2}(0)>0$ and $G_{\omega, t_{0}}^{\prime}(0)>2\left[2 E_{2}(0) G_{\omega, t_{0}}(0)\right]^{1 / 2}$, then the functional $G_{\omega, t_{0}}$ becomes exponentially unbounded.

Proof. This follows the proof of equivalent results of [13, pages 373-385], and so, only brief details are sketched. For the case (a), we select $\omega=-2 E_{2}(0)>$ 0 . Thus, a quadrature implies

$$
G_{\omega, t_{0}}(t) \geq G_{\omega, t_{0}}(0) \exp \left(\frac{G_{\omega, t_{0}}^{\prime}(0) t}{G_{\omega, t_{0}}(0)}\right), \quad t \geq 0 .
$$

We can always select $t_{0}$ large enough that $G_{\omega, t_{0}}^{\prime}(0)>0$ and then (a) follows. The case (b) follows immediately by taking $\omega=0$.

Finally, case (c) follows the calculations of Knops and Payne ([13], see equation (3.6.14)) and the theorem is proved.

REMARK 3.2. In the case that $E_{2}(0)=0$, inequality (3.7) also implies (see Ames and Straughan [1, page 17])

$$
F(t) \leq[F(0)]^{(1-t / T)}[F(T)]^{t / T},
$$

$0 \leq t \leq T$, where $F=G_{0,0}$. Thus, if we consider homogeneous initial conditions, we conclude that the solution is always zero, so we obtain uniqueness of solutions in the thermoelasticity without energy dissipation by using the usual logarithmic convexity argument.

ACKNOWLEdGment. This research is supported by the project "Mathematical Aspects in Generalized thermomechanical theories” BFM 2000-0809.

\section{REFERENCES}

[1] K. A. Ames and B. Straughan, Non-Standard and Improperly Posed Problems, Academic Press, California, 1997.

[2] G. Caviglia, A. Morro, and B. Straughan, Thermoelasticity at cryogenic temperatures, Int. J. Non-Linear Mech. 27 (1992), no. 2, 251-263.

[3] D. S. Chandrasekharaiah, Thermoelasticity with second sound: A review, Appl. Mech. Rev. 39 (1986), 355-376.

[4] - Hyperbolic thermoelasticity: A review of recent literature, Appl. Mech. Rev. 51 (1998), 705-729.

[5] W. Dreyer and H. Struchtrup, Heat pulse experiments revisited, Contin. Mech. Thermodyn. 5 (1993), no. 1, 3-50.

[6] A. E. Green, A note on linear thermoelasticity, Mathematika 19 (1972), 69-75.

[7] A. E. Green and P. M. Naghdi, A re-examination of the basic postulates of thermomechanics, Proc. Roy. Soc. London Ser. A 432 (1991), no. 1885, 171-194.

[8] __ On undamped heat waves in an elastic solid, J. Thermal Stresses 15 (1992), no. 2, 253-264.

[9] _ Thermoelasticity without energy dissipation, J. Elasticity 31 (1993), no. 3, 189-208.

[10] _ A new thermoviscous theory for fluids, J. Non-Newtonian Fluid Mech. 56 (1995), 289-306. 
[11] R. B. Hetnarski and J. Ignaczak, Generalized thermoelasticity, J. Thermal Stresses 22 (1999), no. 4-5, 451-476.

[12] D. Jou, J. Casas-Vázquez, and G. Lebon, Extended Irreversible Thermodynamics, Springer-Verlag, Berlin, 1996.

[13] R. J. Knops and L. E. Payne, Growth estimates for solutions of evolutionary equations in Hilbert space with applications in elastodynamics, Arch. Rational Mech. Anal. 41 (1971), 363-398.

[14] I. Müller and T. Ruggeri, Rational Extended Thermodynamics, Springer Tracts in Natural Philosophy, vol. 37, Springer-Verlag, New York, 1998.

[15] R. Quintanilla, Logarithmic convexity in thermoelasticity of type III, Mathematical and Numerical Aspects of Wave Propagation (Santiago de Compostela, 2000) (A. Bermudez, D. Gomez, C. Hazard, P. Joly, and J. E. Roberts, eds.), SIAM, Pennsylvania, 2000, pp. 192-196.

[16] _ Existence in thermoelasticity without energy dissipation, J. Thermal Stresses 25 (2002), no. 2, 195-202.

[17] R. Quintanilla and B. Straughan, Growth and uniqueness in thermoelasticity, R. Soc. Lond. Proc. Ser. A Math. Phys. Eng. Sci. 456 (2000), no. 1998, 14191429.

Ramón Quintanilla: Departamento Matematica Aplicada 2, Universidad Politecnica de Catalunya, Colon 11, 08222 Terrassa, Barcelona, Spain

E-mail address: ramon@ma2.upc.es 


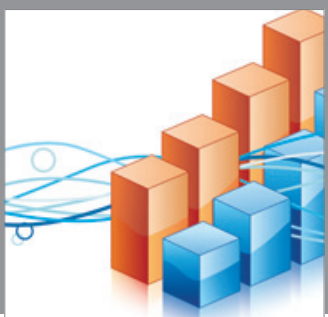

Advances in

Operations Research

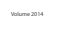

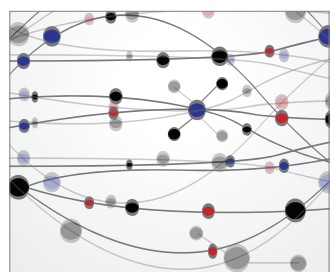

\section{The Scientific} World Journal
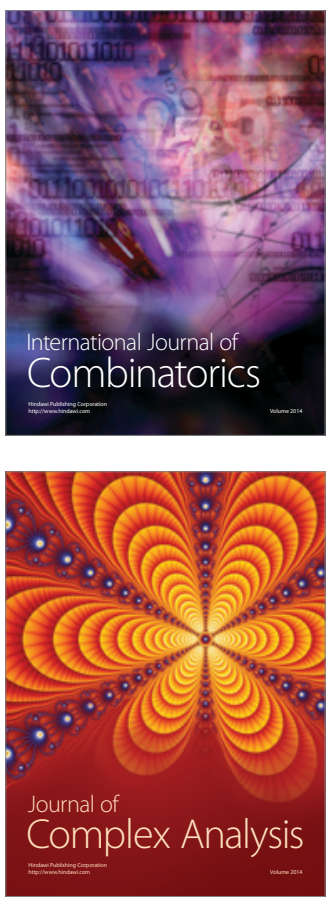

International Journal of

Mathematics and

Mathematical

Sciences
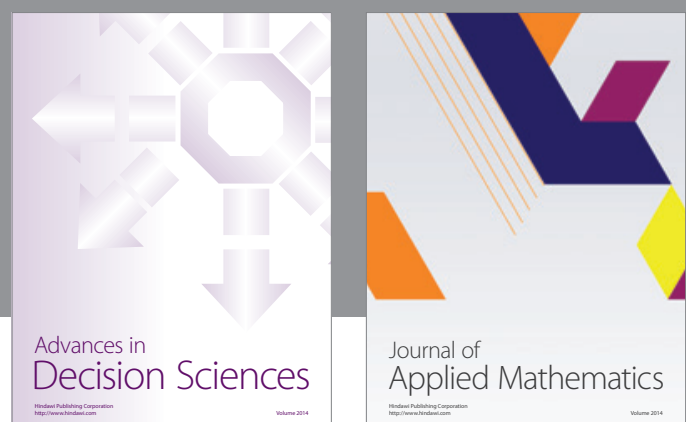

Journal of

Applied Mathematics
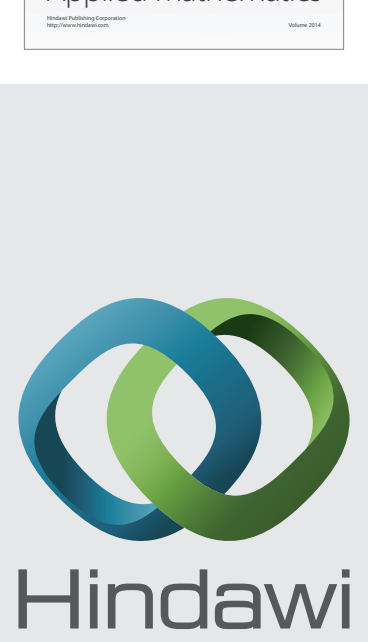

Submit your manuscripts at http://www.hindawi.com
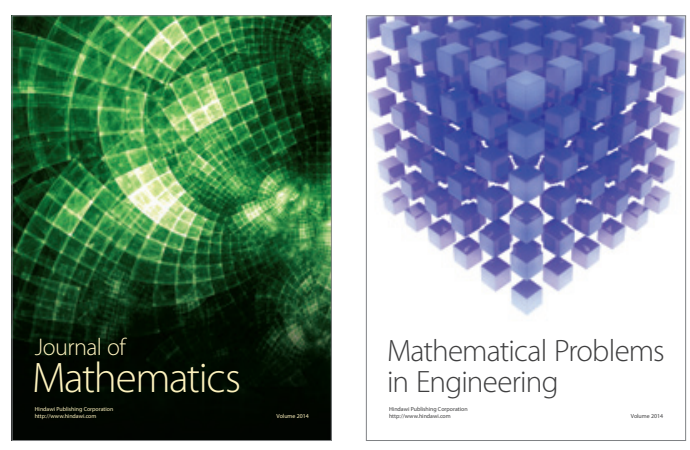

Mathematical Problems in Engineering
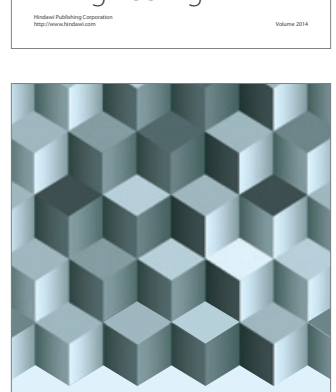

Journal of

Function Spaces
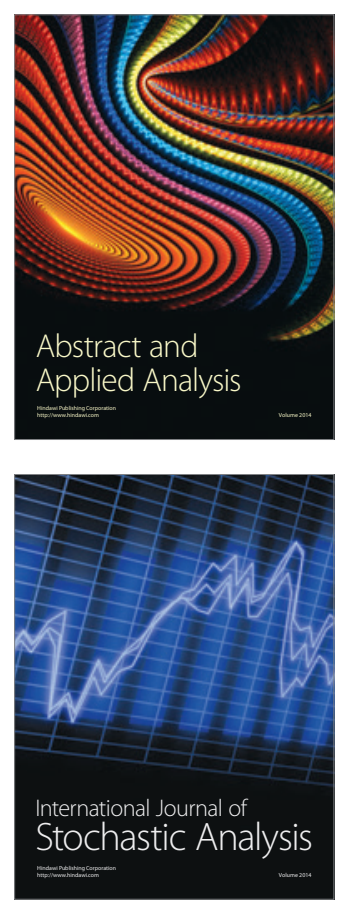

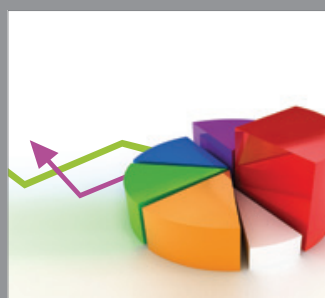

ournal of

Probability and Statistics

Promensencen
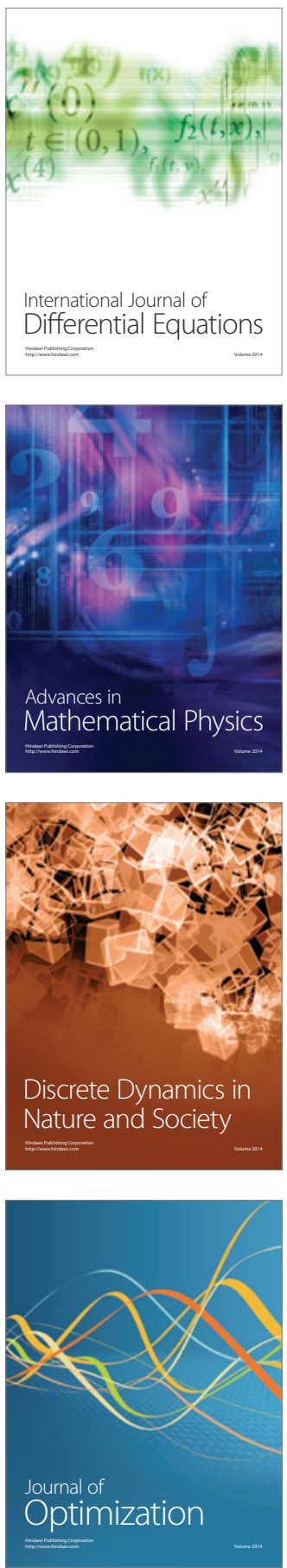